\title{
Determination of Phase Noise Spectra in Optoelectronic Microwave Oscillators: A Langevin Approach
}

\author{
Yanne Kouomou Chembo, Kirill Volyanskiy, Laurent Larger, Enrico Rubiola, and Pere Colet
}

\begin{abstract}
We introduce a stochastic model for the determination of phase noise in optoelectronic oscillators. After a short overview of the main results for the phase diffusion approach in autonomous oscillators, an extension is proposed for the case of optoelectronic oscillators where the microwave is a limit-cycle originated from a bifurcation induced by nonlinearity and time-delay. This Langevin approach based on stochastic calculus is also successfully confronted with experimental measurements.
\end{abstract}

Index Terms-Microwaves, optoelectronic oscillators, phase noise, semiconductor lasers, stochastic analysis.

\section{INTRODUCTION}

$\mathbf{O}$ PTOELECTRONIC oscillators (OEOs) combine a nonlinear modulation of laser light with optical storage to generate ultra-pure microwaves for lightwave telecommunications and radar applications [1], [2]. Their principal specificity is their extremely low phase noise, which can be as low as $-160 \mathrm{dBrad}^{2} / \mathrm{Hz}$ at $10 \mathrm{kHz}$ from a $10 \mathrm{GHz}$ carrier. Despite some interesting preliminary investigations, the theoretical determination of phase noise in OEOs is still a partially unsolved problem. The qualitative features of this phase noise spectrum can be recovered using some heuristical guidelines or rough approximations, but however, a rigorous theoretical background is still lacking.

There are several reasons which can explain that absence of theoretical background. A first reason is that before [3], there was no time domain model to describe such systems, so that stochastic analysis could not be used to perform the phase noise study. Moreover, unlike most of oscillators, the OEO is a delayline oscillator, and very little has been done to study the effect of phase noise on time-delay induced limit-cycles. Finally, the

Manuscript received April 29, 2008; revised June 10, 2008. Current version published January 21, 2009. This work was supported in part by MEC, Spain, and FEDER under Projects TEC2006-10009 (PhoDeCC) and FIS2007-60327 (FISICOS) and in part by ANR project $\mathrm{O}^{2} \mathrm{E}$ under Contract NT05-3_45032. The work of Y. K. Chembo was supported in part by a research fellowship from the Région de Franche-Comté, France.

Y. K. Chembo, K. Volyanskiy, L. Larger, and E. Rubiola are with the Optics Department, Franche Comté Electronique, Mécanique Thermique et Optique-Sciences et Technologies (FEMTO-ST) Institute, 25030 Besançon cedex, France (e-mail: yanne.chembo@femto-st.fr, vol.kirill@gmail.com; laurent.larger@univ-fcomte.fr; rubiola@femto-st.fr).

P. Colet is with the Instituto de Física Interdisciplinar y Sistemas Complejos IFISC (CSIC-UIB), E-07122 Palma de Mallorca, Spain (e-mail: pere@ ifisc.uib. es).

Digital Object Identifier 10.1109/JQE.2008.2002666
OEO is subjected to multiple noise sources, which are sometimes non-white, like the flicker (also referred to as " $1 / f$ ") noise which is predominant around the microwave carrier.

The objective of this work is to propose a theoretical study where all these features are taken into account. The plan of the article is the following. In Section II, we present the phase diffusion approach in autonomous systems. It is a brief review where the fundamental concepts of phase diffusion are recalled, and where some important earlier contributions are highlighted. Then, we derive in Section III a stochastic delay-differential equation for the phase noise study. We show that for our purpose, the global interaction of noise with the system can be decomposed into two contributions, namely an additive and a multiplicative noise contribution. Section IV is devoted to the study of the noise spectrum below threshold. It will appear that the noise power spectrum below threshold is very important for the validation of the stochastic model, but also for an accurate calibration of additive noise. In Section V, we address the problem of phase noise when there is a microwave output using Fourier analysis, and we show that it is possible to have an accurate image of the phase noise spectrum in all frequency ranges. The last section concludes the article.

\section{The Phase Diffusion ApProACh IN Autonomous OSCILLATORS}

\section{A. Fundamental Concepts}

For an ideal (noise-free) oscillator, the Fourier spectrum is a collection of Dirac peaks, standing for the fundamental frequency and its harmonics. The effect of amplitude white noise is to add a flat background, while the peaks do keep their zero linewidth; it is the effect of phase noise to widen the linewidth of these peaks.

Some pioneering papers using stochastic calculus to study the topic of phase noise in autonomous oscillators have been published several decades ago [4]. In particular, it was demonstrated that a general framework to study the problem of phase noise in a self-sustained oscillator could be built on the base of two minimalist assumptions. The first point is that a strong nonlinearity is an essential necessity in oscillators, in the sense that nonlinearity can not be regarded as small because it controls the operating level of the oscillator. The second important point is that the phase is only neutrally stable, so that quasi-linear methods which assume that fluctuations from some operating point are small (linearization techniques) can not be applied directly. 
The phase is neutrally stable as a consequence of the phaseinvariance of autonomous oscillators. In other words, limit-cycles are stable against amplitude perturbations, while there is no mechanism able to stabilize the phase to a given value: hence, phase perturbations are undamped, but they do not diverge exponentially, though. In a noise-free oscillator, the "stroboscopic" state point on the limit-cycle is immobile, but in the presence of noise, it moves randomly along the limit-cycle: in other words, the phase of the oscillator undergoes a diffusion process, in all points similar to a one-dimensional Brownian motion. In the most simple case, the random fluctuations of the phase $\varphi$ are referred to as a Wiener process, obeying an equation of the kind $\dot{\varphi}=\xi(t)$ [with $\varphi(0)=0$ ], where $\xi$ is a Gaussian white noise with autocorrelation $\left\langle\xi(t) \xi\left(t^{\prime}\right)\right\rangle=2 D \delta\left(t-t^{\prime}\right)$, while $D$ is a parameter referred to as the diffusion constant. It can be demonstrated that the phase variance diverges linearly as $\left\langle\varphi^{2}(t)\right\rangle=2 D t$, and the single-side band phase noise spectrum (in $\mathrm{dBc} / \mathrm{Hz}$ ) explicitly reads $\mathcal{L}(\omega)=2 D /\left[D^{2}+\omega^{2}\right]$, so that $D$ is the unique parameter characterizing all the statistical and spectral features of phase fluctuations.

\section{B. The Unifying Theory of Demir, Mehrota and Roychowdhury}

On the base of earlier works by Lax [4] and Kärtner [5], Demir, Mehrota and Roychowdhury have proposed few years ago a unifying theory of phase noise in self-sustained oscillators subjected to white noise sources [6]. Their approach, which had later been extended by Demir to the case of colored noise sources [7], relies on stochastic calculus. The principal point of their contribution was the introduction of a decomposition of phase and amplitude noise through a projection onto the periodic time-varying eigenvectors (the so called Floquet eigenvectors; also see [8]), and they proved that it provides the correct solution to the problem.

Demir et al. have shown that if the sources of noise are Gaussian and white, the phase noise around the fundamental peak (and its harmonics) has a Lorentzian lineshape, and therefore is fully determined by an "effective" diffusion constant $D_{\text {eff }}$ which is the unique parameter needed for phase noise determination. However, if the Demir et al. theory has the great and essential advantage of mathematical rigorousness, its principal drawback is that exactitude is obtained at the expense of simplicity: the calculation of $D_{\text {eff }}$ is very complex, as it requires an accurate determination of all the time-varying eigenvectors related to the autonomous flow. In general this task can only be performed numerically using quite complicated algorithms, and this lack of flexibility explains why this method is scarcely used in the phase noise studies available in the literature. The key challenge for the study of phase noise in OEO would be to provide an accurate description of the phase noise spectrum, while avoiding the determination of Floquet eigenvectors, which is an extremely complicated task in delayed system.

\section{ApPlication OF the Phase Diffusion APPROACH TO OEOS: STOCHASTIC DELAY-DIFFERENTIAL EQUATIONS}

The OEO under study is organized in a single-loop architecture as depicted in Fig. 1. The oscillation loop consists of the following. 1) A wideband integrated optics $\mathrm{LiNbO}_{3}$ Mach-Zehnder (MZ) modulator, seeded by a continuous-wave

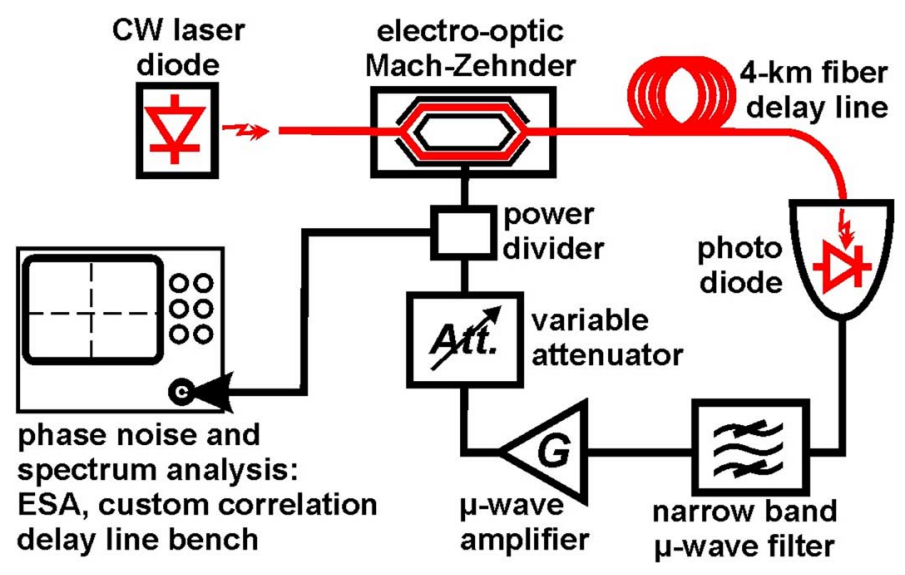

Fig. 1. Experimental setup.

semiconductor laser of optical power $P_{\mathrm{opt}}$; the modulator is characterized by a half-wave voltage $V_{\pi}=4 \mathrm{~V}$. 2) A thermalized $4 \mathrm{~km}$-long fiber performing a time delay of $T=20 \mu \mathrm{s}$ on the microwave signal carried by the optical beam; the corresponding free spectral range is $\Omega_{T} / 2 \pi=1 / T=50$ kHz. 3) A fast photodiode with a conversion factor $S$. 4) A narrow band microwave radio-frequency (RF) filter, of central frequency $F_{0}=\Omega_{0} / 2 \pi=10 \mathrm{GHz}$, and $-3 \mathrm{~dB}$ bandwidth of $\Delta F=\Delta \Omega / 2 \pi=50 \mathrm{MHz}$; 5) A microwave amplifier with gain $G$. 6) A variable attenuator, in order to scan the gain. 7) All optical and electrical losses are gathered in a single attenuation factor $\kappa$.

The dynamics of the microwave oscillation can therefore be described in terms of the dimensionless variable $x(t)=\pi V(t) / 2 V_{\pi}$ whose dynamics obeys [3]

$$
x+\tau \frac{d x}{d t}+\frac{1}{\theta} \int_{t_{0}}^{t} x(s) d s=\beta \cos ^{2}[x(t-T)+\phi]
$$

where $\beta=\pi \kappa \mathrm{SGP}_{\text {opt }} /\left(2 V_{\pi}\right)$ is the normalized loop gain, $\phi=\pi V_{B} /\left(2 V_{\pi}\right)$ is the Mach-Zehnder offset phase, while $\tau=$ $1 / \Delta \Omega$ and $\theta=\Delta \Omega / \Omega_{0}^{2}$ are the characteristic timescale parameters of the bandpass filter. Since we are interested in single-mode microwave oscillations, the solution of (1) can be expressed under the form

$$
x(t)=\frac{1}{2} \mathcal{A}(t) e^{i \Omega_{0} t}+\frac{1}{2} \mathcal{A}^{*}(t) e^{-i \Omega_{0} t}
$$

where $\mathcal{A}(t)=A(t) \exp [i \psi(t)]$ is the slowly varying amplitude of the microwave $x(t)$. We can significantly simplify the right-hand side term of (1) because the cosine of a sinusoidal function of frequency $\Omega_{0}$ can be Fourier-expanded in harmonics of $\Omega_{0}$. In other words, since $x(t)$ is nearly sinusoidal around $\Omega_{0}$, then the Fourier spectrum of $\cos ^{2}[x(t-T)+\phi]$ will be sharply distributed around the harmonics of $\Omega_{0}$ using the relationship $\cos ^{2} z=1 / 2[1+\cos 2 z]$ and the Jacobi-Anger expansion

$$
e^{i z \cos \alpha}=\sum_{n=-\infty}^{+\infty} i^{n} \mathrm{~J}_{\mathrm{n}}(z) e^{i n \alpha}
$$

where $\mathrm{J}_{\mathrm{n}}$ is the $n$th order Bessel function of the first kind. Hence, since the filter of the feedback loop is narrowly resonant around $\Omega_{0}$, it can be demonstrated that discarding all the 
spectral components of the signal except the fundamental is an excellent approximation, so that (1) can be rewritten as

$$
\begin{aligned}
x+\frac{1}{\Delta \Omega} & \frac{d x}{d t}+\frac{\Omega_{0}^{2}}{\Delta \Omega} \int_{t_{0}}^{t} x(s) d s=-\beta \sin 2 \phi \\
& \times \mathrm{J}_{1}[2|\mathcal{A}(t-T)|] \cos \left[\Omega_{0} \cdot(t-T)+\psi(t-T)\right] .
\end{aligned}
$$

In order to include noise effects in this equation, we will consider two main noise contributions in this system.

The first contribution is an additive noise, corresponding to random environmental and internal fluctuations which are uncorrelated from the eventual existence of a microwave signal. The effect of this noise can be accounted for as a Langevin forcing term, to be added in the right-hand side of (4). This additive noise can be assumed to be spectrally white, and since we are interested in its intensity around the carrier frequency $\Omega_{0}$, it can be explicitly written as

$$
\xi_{a}(t)=\frac{1}{2} \zeta_{a}(t) e^{i \Omega_{0} t}+\frac{1}{2} \zeta_{a}^{*}(t) e^{-i \Omega_{0} t}
$$

where $\zeta_{a}(t)$ is a complex Gaussian white noise, whose autocorrelation is $\left\langle\zeta_{a}(t) \zeta_{a}^{*}\left(t^{\prime}\right)\right\rangle=4 D_{a} \delta\left(t-t^{\prime}\right)$, so that the corresponding power density spectrum is $\left|\tilde{\xi}_{a}(\omega)\right|^{2}=2 D_{a}$.

The second contribution is a multiplicative noise due to a noisy loop gain. Effectively, the normalized gain parameter explicitly reads

$$
\gamma=\beta \sin 2 \phi=\frac{\pi}{2} \frac{\kappa \mathrm{SGP}_{\mathrm{opt}}}{V_{\pi}} \sin \left[\pi \frac{V_{B}}{V_{\pi}}\right] .
$$

If all the parameters of the system are noisy [i.e., we replace $\kappa$ by $\kappa+\delta \kappa(t), S$ by $S+\delta S(t)$, etc.], then the gain $\gamma$ may be replaced in (4) by $\gamma+\delta \gamma(t)$, where $\delta \gamma(t)$ is the overall gain fluctuation. We therefore introduce the dimensionless multiplicative noise

$$
\eta_{m}(t)=\frac{\delta \gamma(t)}{\gamma}
$$

which is in fact the relative gain fluctuation. In the OEO configuration, we have $\eta_{m}(t) \ll 1$. This noise is in general spectrally complex, as it is the sum of noise contributions which are very different (noise from the photodetector, from the amplifier, etc.). In agreement with the usual noise spectra of amplifiers and photodetectors, we will here consider that this multiplicative noise is flicker (i.e., varies as $1 / f$ ) near the carrier, and white above a certain knee-value. We therefore assume the following empirical noise power density

$$
\left|\tilde{\eta}_{m}(\omega)\right|^{2}=2 D_{m}\left[1+\frac{\Omega_{H}}{\omega+\Omega_{L}}\right]
$$

where $\Omega_{L}$ is the low corner frequency of the flicker noise, while $\Omega_{H}$ is the high corner frequency. More precisely, we consider that the noise is white below $\Omega_{L}$ and above $\Omega_{H}$, while it remains flicker in between. Typically, we may consider $\Omega_{L} / 2 \pi<1 \mathrm{~Hz}$ and $\Omega_{H} / 2 \pi>10 \mathrm{kHz}$, so that the flicker noise is extended over a frequency span of more than 4 orders of magnitude.
To avoid the integral term of (4) which is complicated to manage analytically, it is mathematically convenient to use the intermediate integral variable

$$
u(t)=\int_{t_{0}}^{t} x(s) d s=\frac{1}{2} \mathcal{B}(t) e^{i \Omega_{0} t}+\frac{1}{2} \mathcal{B}^{*}(t) e^{-i \Omega_{0} t}
$$

which is also nearly sinusoidal with a zero mean value. Using (4), (5) and (7), it can be shown that the slowly-varying amplitude $\mathcal{B}(t)$ obeys the stochastic equation

$$
\begin{aligned}
\{\ddot{\mathcal{B}}+ & \left.\left(\Delta \Omega+2 i \Omega_{0}\right) \dot{\mathcal{B}}+i \Omega_{0} \Delta \Omega \mathcal{B}\right\} e^{i \Omega_{0} t}+\text { c.c. } \\
= & -2 \Delta \Omega \gamma\left[1+\eta_{m}(t)\right]\left[\frac{1}{2} e^{i \Omega_{0} \cdot(t-T)} e^{i \psi_{T}}+\text { c.c. }\right] \\
& \times \mathrm{J}_{1}\left[2\left|\dot{\mathcal{B}}_{T}+i \Omega_{0} \mathcal{B}_{T}\right|\right] \\
& +2 \Delta \Omega\left[\frac{1}{2} \zeta_{a}(t) e^{i \Omega_{0} t}+\text { c.c. }\right]
\end{aligned}
$$

where c.c. stands for the complex conjugate of the preceding term. Since $\mathcal{B}(t)$ is varying at a much slower rate than the carrier frequency $\Omega_{0}$, we can use the slowly varying approximation and assume that $|\ddot{\mathcal{B}}| \ll \Delta \Omega|\dot{\mathcal{B}}|$ and $|\dot{\mathcal{B}}| \ll \Omega_{0}|\mathcal{B}|$ (see [3], [9], [10]). The relationship $x(t)=\dot{u}(t)$ therefore gives $\mathcal{A}=\dot{\mathcal{B}}+i \Omega_{0} \mathcal{B} \simeq i \Omega_{0} \mathcal{B}$, so that we can finally derive from (10) the following stochastic equation for the slowly varying envelope $\mathcal{A}(t)$

$\dot{\mathcal{A}}=-\mu e^{i \vartheta} \mathcal{A}+2 \gamma \mu e^{i \vartheta}\left[1+\eta_{m}(t)\right] \mathrm{Jc}_{1}\left[2\left|\mathcal{A}_{T}\right|\right] \mathcal{A}_{T}+\mu e^{i \vartheta} \zeta_{a}(t)$

where $\mathrm{Jc}_{1}(x)=\mathrm{J}_{1}(x) / x$ is the first order Bessel cardinal function of the first kind. The phase condition has been set to $e^{-i \Omega_{0} T}=-1$, so that the dynamics of interest is restricted to the case $\gamma \geq 0$. The key parameters of this equation are

$$
\mu=\frac{\Delta \Omega / 2}{\sqrt{1+[1 /(2 Q)]^{2}}} \text { and } \vartheta=\arctan \left[\frac{1}{2 Q}\right]
$$

where $Q=\Omega_{0} / \Delta \Omega=200$ is the quality factor of the RF filter. Since $Q \gg 1$, we may simply consider that $\mu \simeq \Delta \Omega / 2$ and $\vartheta \simeq 1 /(2 Q)$. The complex term $\mu e^{i \vartheta}$ is a kind of "filter operator", which can be equated to the half-bandwidth $\Delta \Omega / 2$ when the $Q$-factor of the filter is sufficiently high, as it was done in [3]. It is also noteworthy that in the complex amplitude (11), the initial multiplicative noise remains a real variable, while the additive noise becomes complex.

We had recently shown, in agreement with the experiment, that the OEO has three fundamental regimes for low to moderate feedback gain values (that is, typically for $|\gamma|<3$ ) [3]. For $\gamma<1$, the system does not oscillate and the trivial fixed point is stable; for $1 \leq \gamma<2.31$, the system sustains a pure microwave oscillation, with a constant amplitude, frequency and phase; and at last, for $\gamma \geq 2.31$, the system enters into a regime where the amplitude of the microwave is unstable, and turns to be nonlinearly modulated. We can consider that this phenomenology is still correct as long as $Q \gg 1$. With the aid of the stochastic delay-differential equation ruling the dynamics of $\mathcal{A}$, we may 
now derive analytically the power spectrum density of the oscillator, below and above threshold. However, it should be stressed that in all cases, stochastic variables should be manipulated with respect to the rules of stochastic calculus when an integral/differential transformation is applied to them [11].

\section{Noise Power Density Spectrum Below THRESHOLD $(\gamma<1)$}

In general, no interest is paid to the study of the noise power density spectrum below threshold in OEOs. This lack of interest can be explained by the fact that there is no oscillation in this regime, and the system randomly fluctuates around the trivial equilibrium. However, as we will further see, this regime is particularly interesting because it enables to understand how the noise interacts with the system.

From the stability theory of delay-differential equations with complex coefficients, the deterministic solution of (11) below threshold is the trivial fixed point $\mathcal{A}=0$. After linearization around this solution, (11) can simply be rewritten as

$$
\dot{\mathcal{A}}=-\mu e^{i \vartheta} \mathcal{A}+\gamma \mu e^{i \vartheta} \mathcal{A}_{T}+\mu e^{i \vartheta} \zeta_{a}(t)
$$

where we have used $\mathrm{Jc}_{1}(0)=1 / 2$ (definition by continuity). This equation indicates that the multiplicative noise has no significative influence below threshold, because the product $\eta_{m} \mathcal{A}_{T}$ is a second-order term. Therefore, the noise power below threshold is essentially determined by additive noise.

Equation (13) is linear with constant coefficients: hence, the power density spectrum can be directly obtained as

$$
|\tilde{\mathcal{A}}(\omega)|^{2}=\frac{4 \mu^{2} D_{a}}{\left|i \omega e^{-i \vartheta}+\mu\left(1-\gamma e^{-i \omega T}\right)\right|^{2}} .
$$

One can determine the total output power below threshold due to the white noise fluctuations in the system through the formula

$$
\bar{P}_{\gamma}=\left(\frac{2 V_{\pi}}{\pi}\right)^{2} \frac{\left\langle|\mathcal{A}(t)|^{2}\right\rangle}{2 R_{\text {out }}}
$$

where $R_{\text {out }}$ is the output impedance (in our case, $R_{\text {out }}=50 \Omega$ ), while the brackets and the overbar indicate time-averaging. The dimensionless power $\left\langle|\mathcal{A}(t)|^{2}\right\rangle$ can not be calculated analytically for $\gamma \neq 0$ : it can nevertheless be determined either by numerical simulation of (13), or through a numerical computation of the integral $(1 / 2 \pi) \int_{-\infty}^{+\infty}|\tilde{\mathcal{A}}(\omega)|^{2} d \omega$, where $|\tilde{\mathcal{A}}(\omega)|^{2}$ is given by (14).

However, in the open-loop configuration $(\gamma=0)$, the noisy output power can be analytically determined as

$$
\bar{P}_{0}=\frac{4 V_{\pi}^{2}}{\pi R_{\text {out }}} \Delta F D_{a}
$$

through the use of the Fourier integral, or using fundamental results from stochastic calculus since (13) degenerates to the well-known Orstein-Uhlenbeck equation. Therefore, knowing the bandwidth $\Delta F$ of the RF filter and the half-wave voltage $V_{\pi}$ of the MZ interferometer, an open-loop measurement of the output power can directly give an experimental value for the white noise power density $D_{a}$ through (16).

In our system, we have experimentally measured $\bar{P}_{0}=20.0 \mathrm{nW}$ (or $-47 \mathrm{dBm}$ ), which corresponds to

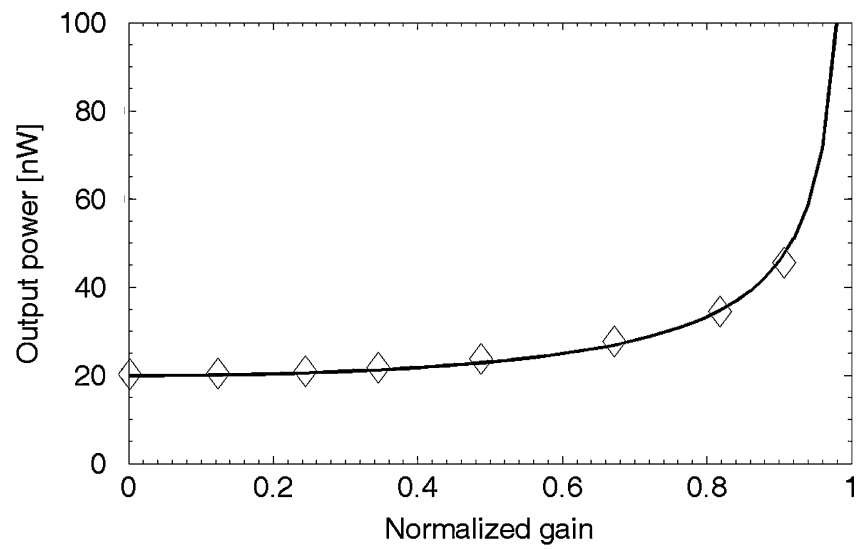

Fig. 2. Variation of the RF noise output power $\bar{P}_{\gamma}$ as a function of the normalized gain, under threshold. The solid line is the theoretical prediction of (15) with $D_{a}=9.8 \times 10^{-16} \mathrm{rad}^{2} / \mathrm{Hz}$, and the symbols represent the experimentally measured data. The gain was varied through attenuation in the electric branch of the loop.

$D_{a}=9.8 \times 10^{-16} \mathrm{rad}^{2} / \mathrm{Hz}$. This value for the power can also be obtained by other means [see Appendix A]. The curve displaying the power variation as a function of the normalized gain under threshold is shown in Fig. 2, and there is an excellent agreement between the experimental data and our analytical formula of (15). It may be interesting to note that the noise power apparently diverges at $\gamma=1$. In fact, one should not forget that this result is obtained using (13), which is only valid for $|\mathcal{A}| \ll 1$. When $\gamma \rightarrow 1$, the amplitude of $\mathcal{A}$ increases and the higher order terms of the Bessel cardinal function are not negligible anymore, so that (13) and (14) are no more valid. Hence, divergence of the noise power is prevented by the nonlinear terms of (11) which become predominant in a very narrow range just below the threshold. A noteworthy study on this topic of noisy oscillators near threshold is [12].

It is also noteworthy that for $\gamma=0$, the noise spectrum follows the spectral shape of the RF filter. However, when $\gamma$ is increased (still below threshold), a first qualitative difference emerges, since the spectrum still follows the spectral shape of the filter, but its fine structure is composed by a collection of peaks which are the signature of microwave ring-cavity modes, as it can be seen in Figs. 3 and 4 .

\section{Phase Noise Spectrum Above Threshold $(\gamma>1)$}

Above threshold, the amplitude of the microwave obeys the nonlinear algebraic equation $\mathrm{Jc}_{1}\left[2\left|\mathcal{A}_{0}\right|\right]=1 /(2 \gamma)$. Using this steady-state relationship, we can neglect the second-order amplitude fluctuations and rewrite (11) under the form

$$
\dot{\mathcal{A}}=-\mu e^{i \vartheta} \mathcal{A}+\mu e^{i \vartheta}\left[1+\eta_{m}(t)\right] \mathcal{A}_{T}+\mu e^{i \vartheta} \zeta_{a}(t) .
$$

We should now look for an equation for the phase $\psi$ in order to find its power density spectrum $|\Psi(\omega)|^{2}$.

Using the Itô rules of stochastic calculus [see Appendix B], we derive the following time-domain equation for the phase dynamics

$$
\dot{\psi}=-\mu\left(\psi-\psi_{T}\right)+\frac{\mu}{2 Q} \eta_{m}(t)+\frac{\mu}{\left|\mathcal{A}_{0}\right|} \xi_{a, \psi}(t)
$$




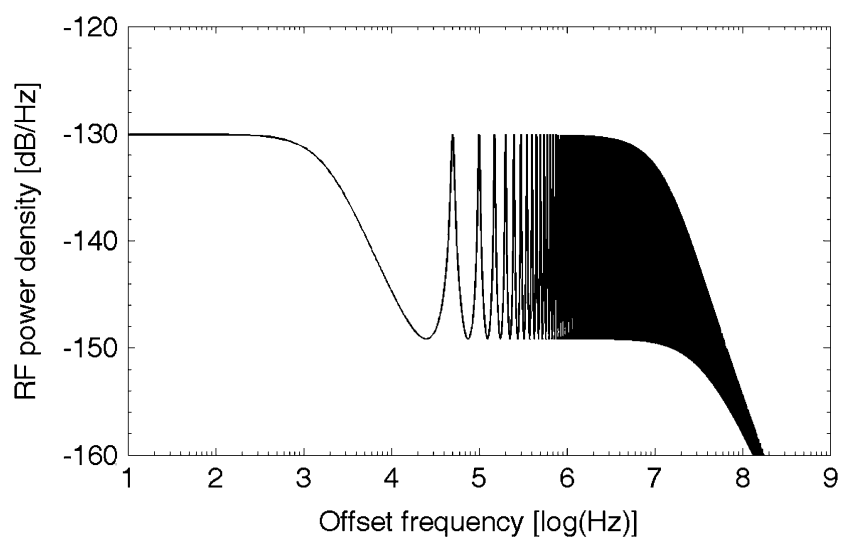

Fig. 3. Theoretical power density spectrum $|\tilde{\mathcal{A}}(f)|^{2}$ of the microwave noise signal below threshold, with $D_{a}=9.8 \times 10^{-16} \mathrm{rad}^{2} / \mathrm{Hz}$ and $\gamma=0.8$, using (14). The semi-logarithmic scale is adopted because it enables to see at the same time the fine structure of regularly spaced ring-cavity peaks, and the global variation shaped by the RF filter bandwidth. This spectrum is divided into two areas: a quasi-flat area within bandwidth, and a $-20 \mathrm{~dB} / \mathrm{dec}$ decrease outside the bandwidth.
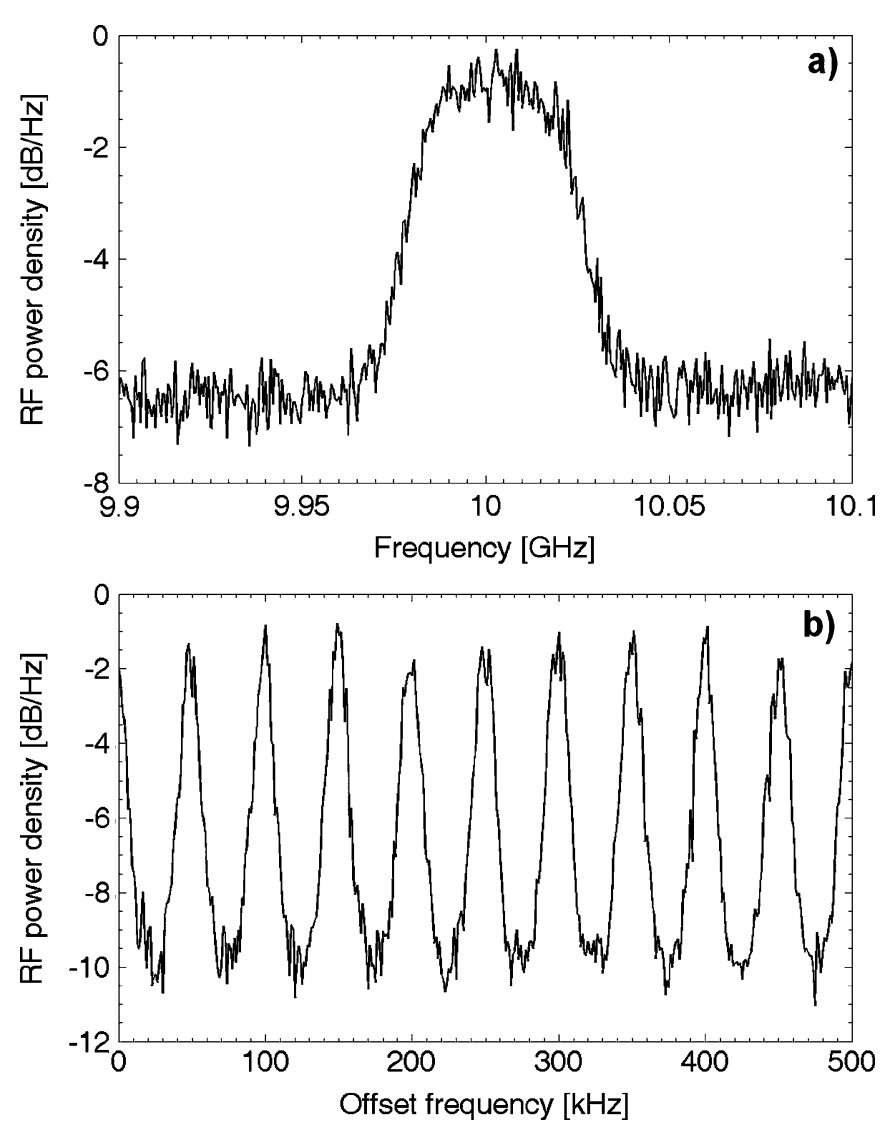

Fig. 4. Experimental power density spectra of the microwave noise below threshold. The spectrum has been scaled to its maximum (reference at $\sim 0 \mathrm{~dB}$ ). (a) Spectrum in a $200 \mathrm{MHz}$ window, showing how the noisy power density is profiled by the RF filter. (b) Zoom-in with near the $10 \mathrm{GHz}$ central frequency in a $500 \mathrm{kHz}$ window, showing the noisy ring-cavity peaks.

where $\xi_{a, \psi}(t)$ is a real Gaussian white noise of correlation $\left\langle\xi_{a, \psi}(t) \xi_{a, \psi}\left(t^{\prime}\right)\right\rangle=2 D_{a} \delta\left(t-t^{\prime}\right)$ [same variance as $\left.\xi_{a}(t)\right]$. We

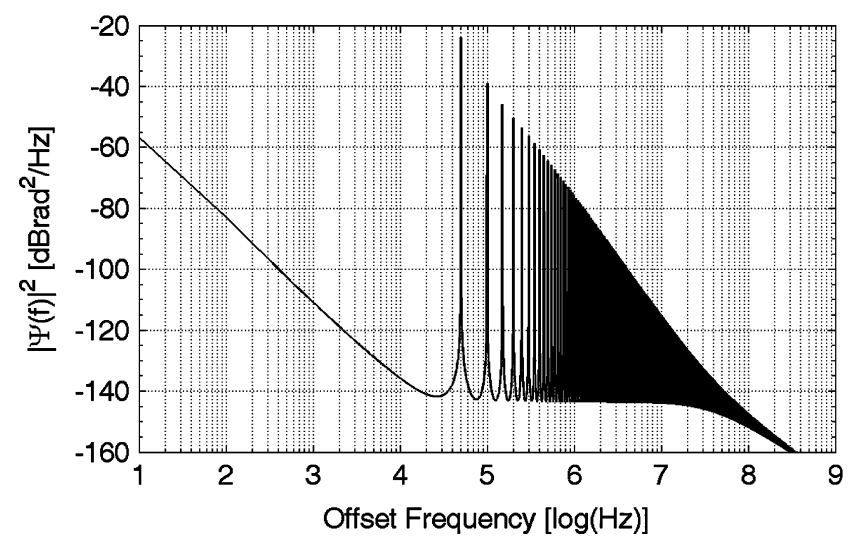

Fig. 5. Theoretical phase noise spectrum above threshold in a $500 \mathrm{MHz}$ window, with $D_{a}=9.8 \times 10^{-16} \mathrm{rad}^{2} / \mathrm{Hz}, D_{m}=5 \times 10^{-11} \mathrm{rad}^{2} / \mathrm{Hz}$, $\Omega_{H}=100 \mathrm{kHz}$ and $\Omega_{L}=1 \mathrm{~Hz}$. The dimensionless amplitude of the microwave oscillation is $\left|\mathcal{A}_{0}\right|=0.41$, corresponding to a power of $10.5 \mathrm{dBm}$.

can use (18) to obtain the Fourier spectrum $\Psi(\omega)$ of the phase $\psi(t)$, and then its power density spectrum following

$$
|\Psi(\omega)|^{2}=\left|\mu \frac{(2 Q)^{-1} \tilde{\eta}_{m}(\omega)+\left|\mathcal{A}_{0}\right|^{-1} \tilde{\xi}_{a, \psi}(\omega)}{i \omega+\mu\left[1-e^{-i \omega T}\right]}\right|^{2} .
$$

Note that here, the influence of gain on phase noise is not explicit anymore: it is implicitly contained in $\left|\mathcal{A}_{0}\right|$. Fig. 5 displays the phase noise spectrum explicitly expressed by (19), and we can now analyze how the spectrum behaves according to the various frequency ranges.

\section{A. Phase Noise Close to the Carrier $\left(\omega<\Omega_{H}\right)$}

Here, we consider the spectrum for frequencies which are relatively close to the carrier (with $\omega>\Omega_{L}$, however). Qualitatively, this corresponds to the frequencies that are much smaller than the high corner value $\Omega_{H}$ of the multiplicative flicker noise. In this region, flicker noise is stronger than white noise, so that $\left|\tilde{\eta}_{m}(\omega)\right| / 2 Q \gg\left|\tilde{\xi}_{a, \psi}(\omega)\right|$. On the other hand, we can also consider that $1-e^{-i \omega T} \simeq i \omega T$. Therefore, taking into account the fact that $\mu T \gg 1,(19)$ can be simplified into

$$
\left|\Psi_{\text {close }}(\omega)\right|^{2} \sim \frac{\left|\tilde{\eta}_{m}(\omega)\right|^{2}}{4 Q^{2} T^{2}} \frac{1}{\omega^{2}} \simeq \frac{\Omega_{H} D_{m}}{2 Q^{2} T^{2}} \frac{1}{\omega^{3}} .
$$

Some remarks can be made at this stage. First, The Leeson effect [13] is here very explicit: the phase noise spectrum decreases as $f^{-3}$ due to the $f^{-1}$ flicker noise. Secondly, phase noise is inversely proportional to $Q^{2}$. Thirdly, phase noise is practically independent of the microwave amplitude $\left|\mathcal{A}_{0}\right|$, as long as multiplicative noise is stronger than additive noise near the carrier. Finally, phase noise decreases as $T^{-2}$, therefore justifying the need for very long delay-lines to reduce phase noise close to the carrier. This dependence was also recovered analytically by Yao and Maleki, using another theoretical approach [1]. Hence, in first approximation there are three ways to reduce phase noise close to the carrier: reduce the power $D_{m}$ of flicker noise, increase the delay $T$ or increase the $Q$ factor of the RF filter. 


\section{B. Phase Noise in the Spurious Peaks Range $\left(\Omega_{H}<\omega \ll \mu\right)$}

The frequencies of concern are here those which are well within the bandwidth, but not too close from the carrier. This range typically lies between $50 \mathrm{kHz}$ and few $\mathrm{MHz}$, and contains the parasite ring-cavity peaks. It is also an area where the multiplicative and additive noises are both white [in the sense that in that range, $\left|\tilde{\eta}_{m}(\omega)\right|^{2}$ and $\left|\tilde{\xi}_{a, \psi}(\omega)\right|^{2}$ are both constant].

The local minima of the spectrum in that area are obtained for $e^{-i \omega T}=-1$, so that the floor of phase noise after the flicker decrease is

$$
\left|\Psi_{\text {floor }}\right|^{2} \simeq \frac{1}{4}\left[\frac{\sqrt{2 D_{m}}}{2 Q}+\frac{\sqrt{2 D_{a}}}{\left|\mathcal{A}_{0}\right|}\right]^{2} \sim \frac{1}{2} \frac{D_{a}}{\left|\mathcal{A}_{0}\right|^{2}}
$$

when $Q$ is sufficiently high. This level is $6 \mathrm{~dB}$ below the additive white noise power density scaled to the power of the microwave. The recipe for a low phase noise floor is then quite simple, and also quite conventional: low additive noise $D_{a}$, and high power $\left|\mathcal{A}_{0}\right|^{2}$ for the microwave signal. The bandwidth does not play any role in this case, as long as the multiplicative noise is not too strong.

The spurious ring-cavity peaks are localized around integer multiples of the round-trip frequency $\Omega_{T} / 2 \pi=1 / T=50 \mathrm{kHz}$. More precisely, a fourth order Taylor expansion of the denominator of (19) shows that around these resonance frequencies, phase noise can be expressed as

$$
\begin{aligned}
& \left|\Psi\left(n \Omega_{T}+\delta \omega\right)\right|^{2} \\
& \quad=\frac{\left.\left.\mu^{2}\left|(2 Q)^{-1} \tilde{\eta}_{m}(\omega)+\right| \mathcal{A}_{0}\right|^{-1} \tilde{\xi}_{a, \psi}(\omega)\right|^{2}}{\left(n \Omega_{T}+\mu T \delta \omega\right)^{2}-\frac{1}{3} n \Omega_{T} \mu T^{3} \delta \omega^{3}-\frac{1}{12} \mu^{2} T^{4} \delta \omega^{4}}
\end{aligned}
$$

where $n$ is the order of the ring-cavity peak. By finding the minima of this Taylor-expanded denominator, it can be shown that the spurious peaks are in fact frequency-shifted according to

$$
f_{n}=\frac{n}{T}-\frac{1}{\pi} \frac{n}{\Delta F T^{2}}=n \times 50 \mathrm{kHz}-n \times 16 \mathrm{~Hz} .
$$

Then, their height relatively to the phase noise floor can also be calculated as

$$
\Delta\left|\Psi_{n}\right|_{\mathrm{dB}}^{2}=10 \log \left[\frac{\Delta F T}{n}\right]^{4}=120 \mathrm{~dB}-40 \log n .
$$

It appears that the level of the spurious peaks increases with the RF bandwidth and with the delay: therefore, a large delay may lead to a lower phase noise near the carrier [see (20)], but it also leads to a higher level for the spurious peaks, so that an optimal trade-off has to be found. It is also noteworthy that this level is independent of the power densities $D_{a}$ or $D_{m}$. In our case, the height of the first spurious peak relatively to the floor is theoretically equal to $120 \mathrm{~dB}$, in excellent agreement with the experimental results of Fig. 6, where a height of $119.5 \mathrm{~dB}$ has been measured. It can also be shown from (22) that the $-3 \mathrm{~dB}$ linewidth of the spurious peaks is

$$
\Delta f_{n}=\frac{2}{\pi} \frac{n^{2}}{\Delta F^{2} T^{3}}=n^{2} \times 32 \mathrm{mHz}
$$
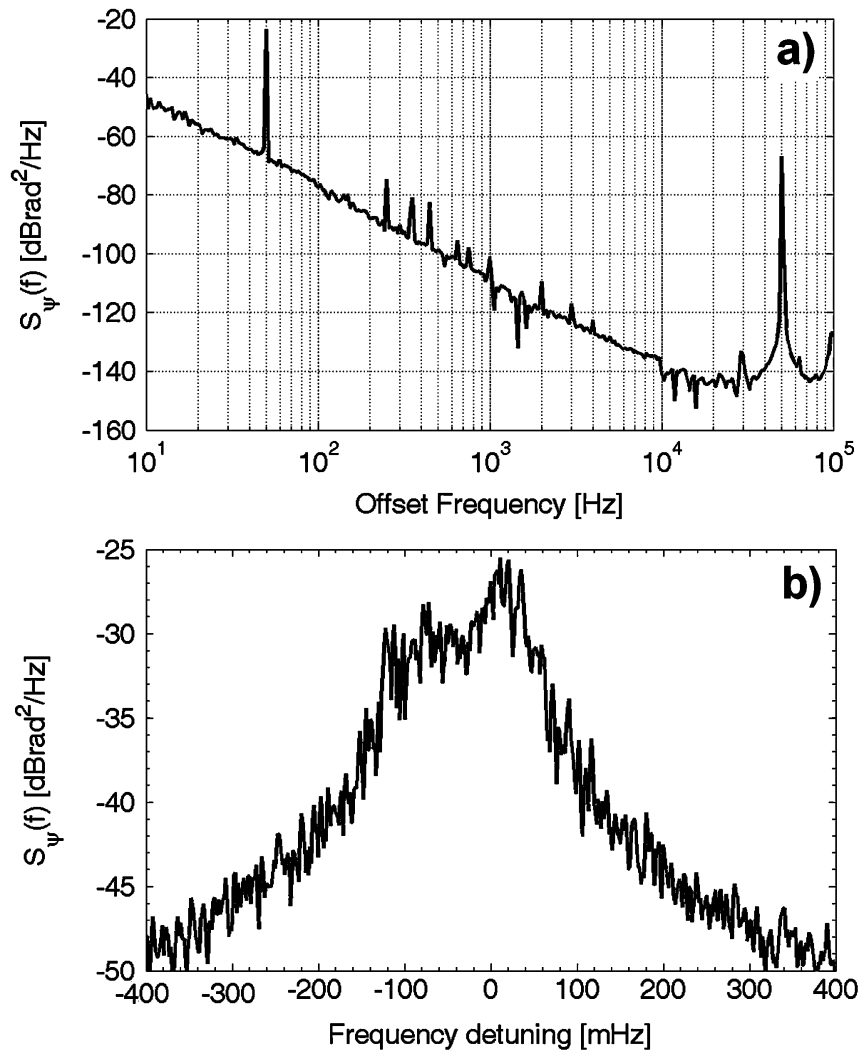

Fig. 6. (a) Experimental phase noise spectrum in a $100 \mathrm{kHz}$ window, showing a noise floor around $-145 \mathrm{dBrad}^{2} / \mathrm{Hz}$ for a microwave power of $10.5 \mathrm{dBm}$. (b) Enlargement of the spectrum around the first spurious peak at the frequency $f_{1}=50594.35 \mathrm{kHz}$. The maximum of this spurious peak is at -25.5 $\mathrm{dBrad}^{2} / \mathrm{Hz}$ [height of the peak: $-119.5 \mathrm{~dB}$ ], and its $-3 \mathrm{~dB}$ linewidth is around $40 \mathrm{mHz}$. All these experimental data are in excellent agreement with the theory. Note that the height of the peak in (a) is not indicative because of insufficient resolution. Also note that the peak at $50 \mathrm{~Hz}$ is a parasite peak originating from the electric mains supply.

an extremely small value which is experimentally confirmed with the results of Fig. 6(b), which displays a linewidth approximately equal to $40 \mathrm{mHz}$ for $n=1$. In comparison, these spurious peaks typically have a linewidth higher than $1 \mathrm{kHz}$ below threshold (see Fig. 3), but their linewidths sharply narrow as $\gamma \rightarrow 1$.

\section{Phase Noise Outside the Bandwidth $(\omega>\mu)$}

Here, the term $\mu\left[1-e^{-i \omega T}\right]$ progressively becomes negligible in the denominator of (19) as $\omega$ is increasing, so that the ringcavity peaks excited by white noise become strongly damped (for being outside the RF bandwidth). In this case, the phase noise decays as

$$
\left|\Psi_{\text {out }}(\omega)\right|^{2} \simeq \frac{2 D_{a} \mu^{2}}{\left|\mathcal{A}_{0}\right|^{2}} \frac{1}{\omega^{2}} \simeq \frac{\Delta \Omega^{2} D_{a}}{2\left|\mathcal{A}_{0}\right|^{2}} \frac{1}{\omega^{2}} .
$$

However, the phase noise does not decrease monotonically as $f^{-2}$ up to infinity: in fact, for $\omega \gg \mu$, there is a second phase noise floor induced by the coupling between phase fluctuations and amplitude fluctuations (second-order effect, see [4]). 


\section{CONCLUSION}

This article has presented a theoretical study of phase noise in OEOs. Our approach has consisted in a Langevin formalism, that is, in adding noise sources to a core deterministic model for the microwave dynamics. We have found an excellent agreement between the main predictions of the model and the experimental results. There is also a good agreement between this theory and the results that are known from the literature, or from our earlier works.

The main advantage of this approach is that it enables within the same framework to understand the behavior of the system under and above threshold, as the same model continuously accounts for all the observed features independently of the value of the gain. It also provides an accurate understanding of phase noise spectra in the whole frequency range. For example, this formalism enables to predict the exact location (that is, the frequency shift) of the spurious microwave ring-cavity peaks, as well as their heights and their linewidths. We have also shown how additive white noise in the system could be related to the open-loop output power, and to the phase noise floor.

However, we have not taken into account in this first model the noise generated by the filter (noisy $\mu$ and $\vartheta$ ), and the delay time (noisy $T$ ). Fluctuations associated to these parameters may induce interesting stochastic features, that will be adressed in future work. Another line of investigation is to achieve a better spectral and statistical fitting of the multiplicative noise $\eta_{m}(t)$, which is an essential variable for the determination of phase noise spectra. Future work will also emphasize on phase noise reduction methods, such as multiple-loop architectures, or quadratic crossed nonlinearities [14]. Finally, this Langevin approach can also be extended to the case of whispering gallery mode OEOs ([15] is a noteworthy reference along that line), and our purpose would therefore be to derive explicit formulas for the corresponding phase noise spectrum.

\section{APPENDIX}

\section{A. Determination of the Output Noise Power for $\gamma=0$}

In the open-loop configuration, the total output power can also be obtained using some quantum electronics formulas.

Effectively, the output power can be explicitly expressed as

$$
\bar{P}_{0}=\frac{1}{2}\left[F k T_{0}+2 e I_{\mathrm{ph}} R_{\mathrm{eq}}\right] G \Delta F
$$

where $G$ is the total gain of our two cascaded amplifiers (22.5 and $22.3 \mathrm{~dB}$ at $10 \mathrm{GHz}$ ), $F=6 \mathrm{~dB}$ is the noise figure of the first amplifier, $T_{0}=295 \mathrm{~K}$ is the room temperature, $k$ is the Boltzmann constant, $e$ is the electron charge, $I_{\mathrm{ph}}=1.2 \mathrm{~mA}$ is the photodiode current, $R_{\mathrm{eq}}=25 \Omega$ is the equivalent load impedance for the photodiode, and $\Delta F=50 \mathrm{MHz}$ is the bandwidth of the RF filter. The formula gives $\bar{P}_{0}=19.6 \mathrm{nW}$, while we have measured $20.0 \mathrm{nW}$. The combination of (16) and (27) therefore gives a method to determine $D_{a}$ following

$$
D_{a}=\frac{\pi R_{\mathrm{out}} G}{8 V_{\pi}^{2}}\left[F k T_{0}+2 e I_{\mathrm{ph}} R_{\mathrm{eq}}\right]
$$

This formula enables to determine without any need for measurements some key performances of the OEO [such as for example the phase noise floor, see (21)] from the specifications of the various optoelectronic components used in the oscillation loop.

\section{B. Derivation of the Stochastic Phase Equation}

We use Itô chain rules to derive the stochastic differential equation for the phase. We first rewrite (17) under the differential form

$$
d \mathcal{A}=-\mu e^{i \vartheta} \mathcal{A} d t+\mu e^{i \vartheta}\left[1+\eta_{m}(t)\right] \mathcal{A}_{T} d t+\mu e^{i \vartheta} d \mathcal{W}_{a}
$$

where $d \mathcal{W}_{a}(t)=d W_{a, r}(t)+i d W_{a, i}(t)$ is a differential Wiener process. Note that the real and imaginary parts of the differential Wiener process are uncorrelated $\left[\left\langle d W_{a, r} d W_{a, i}\right\rangle=0\right]$, have a zero mean value $\left[\left\langle d W_{a, r}\right\rangle=\left\langle d W_{a, i}\right\rangle=0\right]$, and have equal variances $\left[\left\langle\left(d W_{a, r}\right)^{2}\right\rangle=\left\langle\left(d W_{a, i}\right)^{2}\right\rangle=2 D_{a} d t\right]$. The fact that $d W \sim \mathcal{O}(\sqrt{d t}) \gg d t$ explains why the differential terms of second order should be taken into account in stochastic calculus, so that usual differentiation and chain rules do not generally apply. As far as second-order terms are concerned, one may consider $\left(d W_{a}\right)^{2} \approx\left\langle\left(d W_{a}\right)^{2}\right\rangle=2 D_{a} d t$, and discard higherorder terms since $\left\langle\left(d W_{a}\right)^{k+2}\right\rangle \approx \mathcal{O}\left[(d t)^{k}\right] \ll d t$ for $k>0$.

We set $\mathcal{A}(t)=\sqrt{P(t)} e^{i \psi(t)}=e^{\rho(t)+i \psi(t)}$, where $\rho=$ $(1 / 2) \ln P$ is an auxiliary variable. At order $d t$, we have

$$
\begin{aligned}
d \rho+i d \psi & =d \ln \mathcal{A} \\
& =\frac{d \mathcal{A}}{\mathcal{A}}-\frac{1}{2}\left[\frac{d \mathcal{A}}{\mathcal{A}}\right]^{2} \\
& =-\mu e^{i \vartheta} d t+\mu e^{i \vartheta}\left[1+\eta_{m}(t)\right] \frac{\mathcal{A}_{T}}{\mathcal{A}} d t+\frac{\mu e^{i \vartheta}}{\mathcal{A}} d \mathcal{W}_{a} .
\end{aligned}
$$

Note that from the definition of the differential Wiener process $d \mathcal{W}_{a}(t)$ given just below (29), we can consider that $\left|d \mathcal{W}_{a}\right|^{2} \approx$ $\left\langle\left|d \mathcal{W}_{a}\right|^{2}\right\rangle=4 D_{a} d t$ and $\left(d \mathcal{W}_{a}\right)^{2} \approx\left\langle\left(d \mathcal{W}_{a}\right)^{2}\right\rangle=0$. Assuming second-order fluctuations for the amplitude (that is, $\left|\mathcal{A}_{T}\right| \simeq$ $|\mathcal{A}|)$, we are led to

$$
\begin{array}{r}
d \psi=-\mu \sin \vartheta d t+\mu\left[1+\eta_{m}(t)\right] \sin [\vartheta \\
\left.+\psi_{T}-\psi\right] d t \\
+\frac{\mu}{\left|\mathcal{A}_{0}\right|} d W_{a, \psi}
\end{array}
$$

where $d W_{a, \psi}=d W_{a, r} \sin \vartheta+d W_{a, i} \cos \vartheta$ is a real Gaussian white noise with zero mean and variance $\left\langle\left(d W_{a, \psi}\right)^{2}\right\rangle=2 D_{a} d t$. Since $\left|\psi-\psi_{T}\right| \ll \vartheta \ll 1$, we have $\sin \left[\vartheta+\psi_{T}-\psi\right] \simeq$ $\vartheta-\left(\psi-\psi_{T}\right)$ so that finally

$$
\dot{\psi}=-\mu\left(\psi-\psi_{T}\right)+\frac{\mu}{2 Q} \eta_{m}(t)+\frac{\mu}{\left|\mathcal{A}_{0}\right|} \xi_{a, \psi}(t) .
$$

This result is also the one we may have recovered through the usual rules of differential calculus (however, it is not so for the equation ruling the power variable $P$ ). Note that this equation is valid only as long as the approximation of neglecting $\ddot{\mathcal{B}}$ in (10) is valid. Also note that the effective phase diffusion coefficients associated to the additive and multiplicative noises are equal 


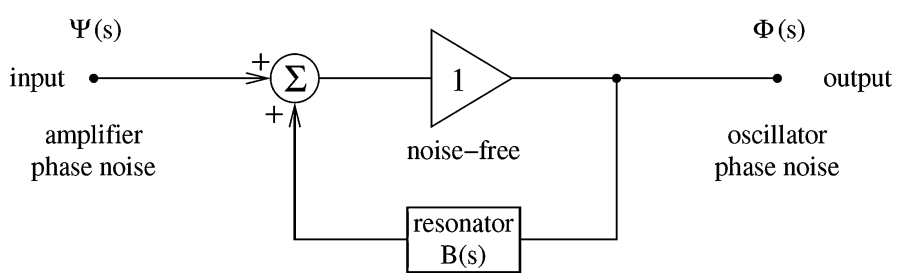

Fig. 7. Oscillator phase noise transfer function (with $s \equiv j f$ ).

to $2 D_{a} \mu^{2} /(2 Q)^{2}$ and $2 D_{m} \mu^{2} /\left|\mathcal{A}_{0}\right|^{2}$ respectively (in the white noise frequency range).

\section{An Alternative Paradigm for Phase Noise Analysis}

It is possible to gain a different physical insight into the phase noise problem in OEOs, using an alternative methodology related to the conventional theory of feedback oscillators. We hereafter briefly sketch the main lines of this heuristical approach.

The oscillator consists of an amplifier of gain $A$ (constant) and of a feedback path of transfer function $\beta(j f)$ in closed loop. The function $\beta(j f)$ selects the oscillation frequency, while the gain $A$ compensates for the feedback loss. This general model is independent of the nature of the amplifier and of the frequency selector. We assume that the Barkhausen condition $|A \beta(j f)|=$ 1 for stationary oscillation is verified at the carrier frequency $f_{0}$ through a gain-control mechanism. Under this hypothesis, the phase noise is modeled by the scheme shown in Fig. 7, in which all signals are the phases of the oscillator loop [16]. The main reason for describing the oscillator in this way is that we get rid of the non-linearity, pushing it in the loop-gain stabilization. The ideal amplifier "repeats" the phase of the input, as it has a gain exactly equal to 1 in the phase noise model. The real amplifier introduces the random phase $\psi(t) \leftrightarrow \Psi(j f)$ in the loop. In this representation, the phase noise is always additive noise, regardless of the physical mechanism involved. This eliminates the mathematical difficulty inherent in the parametric nature of flicker noise and of the noise originated from the environmental fluctuations.

The feedback path is described by the transfer function $\mathrm{B}(j f)$ of the phase perturbation. In the case of the delay-line oscillator, the feedback path is a delay line of delay $T$ followed by a selector filter. The latter is necessary, otherwise the oscillator would oscillate at any frequency multiple of $1 / T$, with no preference. Implementing the selector as a bandpass filter (a resonator) of group delay $T_{g}$, the phase-perturbation response of the feedback path is

$$
\mathrm{B}(j f)=\frac{\exp (-j 2 \pi f T)}{1+j 2 \pi f T_{g}} .
$$

We assume that all the phase perturbations in the loop are collected in the random function $\psi(t) \leftrightarrow \Psi(j f)$, regardless of the physical origin (amplifier, photodetector, optical fiber, etc.). Denoting with $\varphi(t) \leftrightarrow \Phi(j f)$ the oscillator output phase, the oscillator is described by the phase-perturbation transfer function $\mathrm{H}(j f)=\Phi(j f) / \Psi(j f)$. By inspection on Fig. 7, and using the basic equations of feedback, the oscillator transfer function reads

$$
\mathrm{H}(j f)=\frac{1}{1-\mathrm{B}(j f)}
$$

and the oscillator phase noise spectrum would be given by $S_{\varphi}(f)=|\mathrm{H}(j f)|^{2} S_{\psi}(f)$.

\section{REFERENCES}

[1] X. S. Yao and L. Maleki, "Optoelectronic microwave oscillator," J. Opt. Soc. Amer. B, vol. 13, pp. 1725-1735, 1996.

[2] X. S. Yao and L. Maleki, "Optoelectronic oscillator for photonic systems," IEEE J. Quantum Electron., vol. 32, pp. 1141-1149, 1996.

[3] Y. K. Chembo, L. Larger, H. Tavernier, R. Bendoula, E. Rubiola, and P. Colet, "Dynamic instabilities of microwaves generated with optoelectronic oscillators," Opt. Lett., vol. 32, pp. 2571-2573, 2007.

[4] M. Lax, "Classical noise. V. Noise in self-sustained oscillators," Phys. Rev., vol. 160, pp. 290-307, 1967.

[5] F. K. Kärtner, "Analysis of white and $f^{-\alpha}$ noise in oscillators," Int. $J$. Circuit Theory Appl., vol. 18, no. 5, pp. 485-519, May 1990.

[6] A. Demir, A. Mehrotra, and J. Roychowdhury, "Phase noise in oscillators: A unifying theory and numerical methods for characterization," IEEE Trans. Circuits Syst. I, Fund. Theory Appl., vol. 47, no. 5, pp. 655-674, May 2000.

[7] A. Demir, "Phase noise and timing jitter in oscillators with colorednoise sources," IEEE Trans. Circ. Syst. I, Fund. Theory and Appl., vol. 49, pp. 1782-1791, 2002.

[8] G. J. Coram, "A simple 2-D oscillator to determine the correct decomposition of perturbations into amplitude and phase noise," IEEE Trans. Circ. Syst. I, Fund. Theory and Appl., vol. 48, pp. 896-898, 2001.

[9] Y. K. Chembo, L. Larger, R. Bendoula, and P. Colet, "Effects of gain and bandwidth on the multimode behavior of optoelectronic microwave oscillators," Opt. Exp., vol. 16, pp. 9067-9072, 2008.

[10] Y. K. Chembo, L. Larger, and P. Colet, "Nonlinear dynamics and spectral stability of optoelectronic microwave oscillators," IEEE J. Quantum Electron., vol. 44, no. 9, pp. 858-866, Sep. 2008.

[11] M. Lax, "Classical noise. IV: Langevin methods," Rev. Mod. Phys., vol. 38, pp. 541-566, 1966.

[12] R. D. Hempstead and M. Lax, "Classical noise. VI. Noise in self-sustained oscillators near threshold," Phys. Rev., vol. 161, pp. 350-366, 1967.

[13] D. B. Leeson, "A simple model of feedback oscillator noise spectrum," Proc. IEEE, vol. 54, no. 2, pp. 329-330, Feb. 1966.

[14] Y. Pomeau, "Diffusion de phase d'un autooscillateur avec effet de retard," C. R. Acad. Sci. Paris, vol. 310-II, pp. 1025-1029, 1990.

[15] A. B. Matsko, L. Maleki, A. A. Savchenkov, and V. S. Ilchenko, "Whispering gallery mode based optoelectronic microwave oscillator," J. Mod. Opt., vol. 50, pp. 2523-2542, 2003.

[16] E. Rubiola, Phase Noise and Frequency Stability in Oscillators. Cambridge, U.K.: Cambridge Univ. Press, 2008.

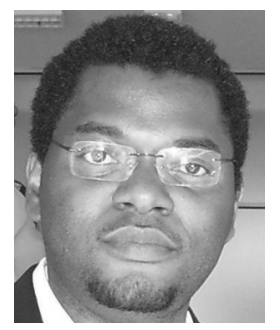

Y. Kouomou Chembo was born in 1976 at Blanc-Mesnil, France. He received the M.Sc. Degree in physics from the University of Yaoundé I, Yaoundé, Cameroon, and a Diploma of Communication Engineering from the National Advanced School of Telecommunications, Yaoundé, both in 2001. He received the Ph.D. degree in physics from the University of Yaoundé I in 2005 and the $\mathrm{Ph} . \mathrm{D}$. degree in laser physics from the Institute for Cross-Disciplinary Physics and Complex Systems (formerly Mediterranean Institute for Advanced Studies), Palma de Mallorca, Spain, in 2006

In 2002, he was with the Division of Technical Telecommunication Studies, Minpostel, Cameroon. He is now a Postdoctoral Fellow at the Franche Comté Electronique, Mécanique Thermique et Optique-Sciences et Technologies (FEMTO-ST) Institute, Besançon, France. His research interests involve nonlinear dynamics, chaos cryptography, optical fiber communications, microwave photonics and semiconductor laser physics. 


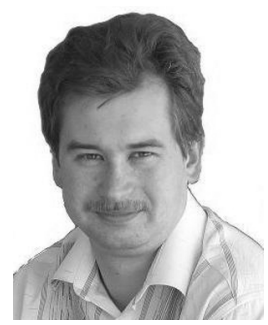

Kirill I. Volyanskiy was born in 1973 . He received the degree in radio-engineering from the St. Petersburg State University of Aerospace Instrumentation, Russia, in 1996. He is currently working toward the $\mathrm{Ph} . \mathrm{D}$. degree on optoelectronic oscillators at the Optics Laboratory of the Franche-Comte University, Besançon, France.

Since 1999, he has been working as a Research and Teaching Assistant with St. Petersburg State University of Aerospace Instrumentation. He engaged research on surface acoustic wave (SAW) devices, particularly in SAW physics using quantum mechanics for the determination of crystal elastic constants, and in SAW device development (chirp filters, torque sensors and so on).

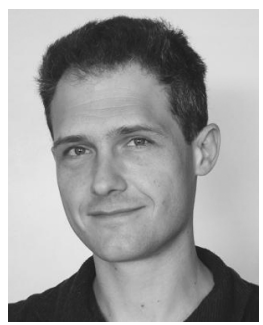

Laurent Larger was born in Colmar, France, in 1968. He received the degree in electronic engineering from the University of Paris XI, Orsay, France, in 1988, the Agrégation degree in applied physics in 1991, and the Ph.D. degree in optical engineering and the "Habilitation" degree from the University of Franche-Comté in 1997 and 2002, respectively.

He was responsible from 2003 to 2006 of the international research center GTL-CNRS Telecom, a joint laboratory between the French CNRS, Georgia Tech University of Atlanta, and the University of Franche-Comté. He got a full professor position at the University of Franche-Comté in 2005. His current research at the Franche Comté Electronique, Mécanique Thermique et Optique-Sciences et Technologies (FEMTO-ST) Institute, Besançon, includes the study of chaos in optical and electronic systems for secure communications, delayed nonlinear dynamics, optical telecommunication systems, and high spectral purity optoelectronic oscillators. Since September 2007 he is a Junior member at the Institut Universitaire de France (IUF).

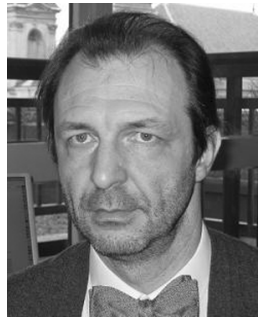

Enrico Rubiola (M'04) received the M.S. degree in electronic engineering from the Politecnico di Torino, Turin, Italy, in 1983, the Ph.D. degree in metrology from the Italian Ministry of Scientific Research, Rome, Italy, in 1989, and the D.Sc. degree from the Université de Franche Comté (UFC), Besançon, France, in 1999.

$\mathrm{He}$ is a currently a Professor with the UFC, and a Scientist with the Franche Comté Electronique, Mécanique Thermique et Optique-Sciences et Technologies (FEMTO-ST) Institute, Besançon. Prior to joining the UFC, he was a Researcher with the Politecnico di Torino, a Professor with the University of Parma, Parma, Italy, and a Professor with the Université Henri Poincaré, Nancy, France. He has been involved with various topics of electronics and metrology, namely, navigation systems, time and frequency comparisons, atomic frequency standards, and gravity. His main fields of interest are precision electronics, phase-noise metrology, frequency synthesis, and low-noise microwave and photonic oscillators.

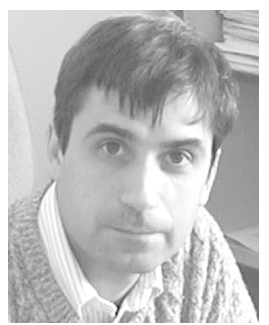

Pere Colet was born in Vilafranca del Penedés, Barcelona, Spain, on April 21, 1964. He received the M.Sc. degree in physics in 1987 from the Universitat de Barcelona, and the Ph.D. degree in physics in 1991 from the Universitat de les Illes Balears, Palma de Mallorca, Spain.

In 1991 he became a Teaching Assistant at the Departament de Física of the Universitat de les Illes Balears. From September 1991 to February 1993 and from April to September 1994 he was a postdoctoral Fulbright fellow at the School of Physics of the Georgia Institute of Tecnology, Atlanta. In October 1994 he joined the Departament de Física of the Universitat de les Illes Balears. Since May 1995 he has a permanent research position at the Spanish Consejo Superior de Investigaciones Cientificas. His present position is Research Professor at IFISC in Palma de Mallorca. He has co-authored over 80 papers in international journals as well as 25 other scientific publications. His research interests include fluctuations and nonlinear dynamics of semiconductor lasers, synchronization of chaotic lasers and encoded communications, synchronization of coupled nonlinear oscillators, pattern formation and quantum fluctuations in nonlinear optical cavities and dynamics of localized structures. 